\title{
ZONAL HARMONICS OF THE GRAVITY FIELD
}

IN DEF-VARIABLES

\section{IGNACIO APARICIO AND LUIS FLORIA}

Grupo de Mecánica Celeste I, Departamento de Matemática Aplicada a la Ingeniería, ETSII. Universidad de Valladolid. E - 47011 Valladolid, Spain.

To take advantage of the linear and regular formulation and treatment of Celestial Mechanics problems (Kustaanheimo \& Stiefel 1965; Stiefel \& Scheifele 1971; Deprit, Elipe \& Ferrer 1994), Sharaf \& Saad (1997) have given an analytical expansion of the Earth's gravitational zonal potential in terms of Kustaanheimo-Stiefel (KS) regular elements (Stiefel \& Scheifele 1971, §19), with special emphasis on its application to elliptic-type two-body orbits and, consequently, using a generalized (elliptic) eccentric anomaly as the independent variable.

Motivated by these and other considerations based on the definition and use of KS elements, and following a treatment similar to that of Stiefel \& Scheifele (1971, §19), we develop element equations corresponding to a $D E F$-formulation of the satellite problem under the effect of the zonal potential.

The weakly canonical DEF-transformation to focal-type variables (Deprit, Elipe \& Ferrer 1994, $\S \S 4.1$ ) was originally devised to exactly linearize the equations of motion governing the conservative, spatial Kepler problem, giving it the form of a 4-dimensional harmonic oscillator. By analogy with Stiefel \& Scheifele (1971, $\S 19)$, the DEF-elements of the motion will be constants of integration occurring in the general solution of the unperturbed harmonic oscillator equations generated by the pure Kepler problem in DEF-variables, whereas for the perturbed problem they satisfy a system of first-order differential equations. Elements vary almost linearly if the motion is subjected to weak perturbations. The equations of motion corresponding to the perturbed problem can be treated by the method of variation of constants, which leads to the said system of differential equations for these quantities (with a true-like anomaly as the independent variable). In certain cases, these equations must also be supplemented by the equations for the variation of other dynamical quantities (e.g., the law of variation of the angular momentum, or the variation of the energy) and by the differential time transformation. All these relations constitute the element equations.

We carry out an application of the focal method (Burdet 1969, §2) to derive the analytical expression, in terms of elements attached to the linearizing DEFvariables, of any zonal harmonic of the gravitational field created by a central body, and obtain the corresponding equations of motion for any value of the eccentricity. To this end, we will follow a variant of the focal method canonical approach based on the (weakly) canonical extension of the projective-decomposition pointtransformation proposed by Deprit et al.

The focal method enjoys the property that the reciprocal of the distance (the quantity $1 / r)$ is represented by a simple polynomial expression in trigonometric 
functions of the true-like anomaly, which facilitates the expansion of negative powers of the distance in the form of Fourier polynomials in that independent variable. Accordingly, our expression for any zonal harmonic turns out to be simpler than those (truncated, but in principle infinite) proposed by Sharaf \& Saad $(1997, \S 3)$ in terms of an eccentric-like anomaly. As a consequence, we expand the zonal harmonics as Fourier polynomials in the true-like anomaly with coefficients depending on the oscillator DEF-elements, the orbital eccentricity and semi-latus rectum. Our description is not restricted to the case of elliptic-type orbits, and so our developments and results are uniformly valid for any type of two-body conic-section orbit.

Research partially supported by Junta de Castilla y León (Grant VA61/98).

\section{References}

Burdet, C. A.: 1969, J. reine angew. Math., 238, 71-84.

Deprit, A., Elipe, A. \& Ferrer, S.: 1994, Celest. Mech. and Dyn. Astron., 58, 151-201.

Kustaanheimo, P., \& Stiefel, E. L.: 1965, J. reine angew. Math., 218, 204-219.

Sharaf, M. A. \& Saad, A. S.: 1997, Celest. Mech. and Dyn. Astron., 66, 181-190.

Stiefel, E. L. \& Scheifele, G.: 1971, "Linear and Regular Celestial Mechanics.", Springer-Verlag, Berlin-Heidelberg-New York. 